\title{
Deuten aus der Perspektive der strukturalen Psychoanalyse Lacans
}

\author{
Manfred Klemann
}

Online publiziert: 31 . Oktober 2013

(C) Die Autor(en) 2013. Dieser Artikel ist auf Springerlink.com mit Open Access verfügbar.

Zusammenfassung Im vorliegenden Beitrag wird der Zusammenhang zwischen Verstehen und Gegenübertragungsanalyse im Hinblick auf den analytischen Deutungsprozess untersucht. Darin spielt das Selbstverständnis des Analytikers eine entscheidende Rolle im Hinblick darauf, welche Richtung eine Analyse nimmt. Da Feld und Funktion des Sprechens dabei entscheidend sind, werden sie kurz skizziert, um den struktural-analytischen Ausgangspunkt der nachfolgenden Überlegungen verständlich zu machen. Ausgangspunkt der weiteren Überlegung ist die Definition des Unbewussten als das, was einem nicht bewusst zur Verfügung steht. Wenn sich jedoch der Analytiker mit dem Platz des Wissenden identifiziert, auf den ihn die Übertragung des Analysanden setzt, resultiert daraus eine Deutungsgewissheit, welche die Analyse zwangsläufig in eine Sackgasse führt. Dies ist typisch für die forcierte Übertragungsanalyse im Hier und Jetzt. Dem wird als Alternative die Technik des Andeutens gegenübergestellt, deren konzeptueller Ausgangspunkt gerade das Nichtverstehen (i. S. von Nichtwissen) ist. Diese Annahme schafft die Voraussetzung, sich weniger auf die Analyse der Gegenübertragung stützen zu müssen. Stattdessen rückt das Gesprochene ins Zentrum der Wahrnehmung und die darin befindlichen Brüche. Eine solche analytische Haltung ermöglicht es, das Sprechen des Subjekts des Unbewussten durch den Umweg über den Analytiker und dessen An-Deutungen zu hören.

Dr. phil., Dipl.-Psych. M. Klemann ( $₫)$ Goetheallee 8, 37073 Göttingen, Deutschland E-Mail: dr.manfred.klemann@t-online.de 


\title{
Interpreting from the perspective of structural Lacanian psychoanalysis
}

\begin{abstract}
Beware of understanding!" Lacan's structural analysis: theory and practice of interpretation. This paper investigates the relationship of understanding and countertransference analysis in the process of analytical interpretation. Here selfimage of the analyst is crucial concerning the direction of the analysis. As field and function of speech are pivotal, they will be briefly sketched to clarify the structuralanalytic starting point of the following arguments. The basis of the ideas is a definition of the unconscious as what is not conscious available. Should the analyst see himself as the "knowing" one, as his patient also assumes, a false assurance will result and the analysis will lead nowhere. This is typical of a "forced" transference analysis in the here and now. The alternative is a strategy of hints, whose premise is, that we do not know. This assumption makes it unnecessary to rely on countertransference analysis. Instead, the spoken word and its contradictions are in the focus. This analytical attitude makes listening to the "subject of the unconscious" possible by means of the analyst and his hints.
\end{abstract}

Hüten Sie sich, zu verstehen!

\section{Verstehen als Zentralbegriff des psychoanalytischen Diskurses}

Das Motto des Aufsatzes kolportiert eine Äußerung Lacans aus dem Jahr 1954 [1991]. Isoliert betrachtet ist sie auf den ersten Blick irritierend, weil Verstehen zu den Zentralbegriffen des modernen psychoanalytischen Diskurses gehört. Im Kontext der sog. Gegenübertragungsanalyse nimmt das Verstehen den zentralen Dreh- und Angelpunkt ein, von dem aus das Unbewusste des Patienten unmittelbar zugänglich erscheint. Dem liegt die Freudsche Metapher vom Receiver und Teller (Freud 1912e) zugrunde, die im neurobiologischen Diskurs vom Konzept der Spiegelneurone abgelöst worden ist. Beiden Konzepten ist gemeinsam, dass die Frage, wie in der Psychoanalyse gedeutet wird, konzeptuell nicht geklärt, sondern im Imaginären verbleibt.

Wenn Einigkeit darüber besteht, dass das Unbewusste per definitionem das umfasst, was unbewusst, also dem Bewusstsein nicht zugänglich, sondern ihm vielmehr fremd ist, dann kann ein Verstehen seelischer unbewusster Vorgänge nicht am Beginn einer analytischen Begegnung, sondern höchstens am Ende stehen. So, wie sich auch die Bedeutung eines Satzes frühestens dann erschließt, wenn das letzte Wort gesprochen und der Zusammenhang, in dem er steht, mit berücksichtigt wird.

Die vorliegende Arbeit versucht der Frage nachzugehen, wie sich die Themenkreise ,Verstehen“ - als vorbereitender Akt einer Deutung - und ,das Selbstverständnis des Analytikers“ miteinander verschränken. Dabei wird es auch um die Frage gehen, was es eigentlich heißt, psychoanalytisch zu deuten und welche Probleme damit verbunden sein können. 
Detaillierte etymologische Untersuchungen $\mathrm{zu}$ den Begriffen Verstehen und Deuten würden den Rahmen dieser Arbeit sprengen. Soviel sei immerhin gesagt, dass es um die Sinnfrage geht; allerdings nicht nur aus einer hermeneutischen, sondern auch aus einer philosophisch-praktischen Perspektive. Für die hier entwickelten Überlegungen sind besonders die Bereiche Sprache und Sprechen von Interesse, weil diese das psychoanalytische Arbeits- und Untersuchungsfeld abstecken.

\section{Zur Theorie des Deutens}

Von der Sprache und vom Sprechen

Der Sinn sprachlicher Äußerungen ergibt sich aus Verweisen, d. h. Worte beziehen sich auf andere Worte. Oder psychoanalytisch gesprochen: Worte sind miteinander assoziiert. Die daraus resultierenden Wortketten oder Assoziationen sind der wesentliche Grund dafür, dass Gesprochenes vieldeutig ist. Da Worte der Alltagssprache zusätzlich von subjektiven Bedeutungsaspekten eingefärbt sind und somit einen subjektiven Sinn enthalten, von dem der jeweilige Sprecher bewusst oder unbewusst annimmt, dass er dem Hörer selbstverständlich bekannt und vertraut ist, erhöht sich die Komplexität der Kommunikation erheblich.

Die psychoanalytische Ausgangsposition besteht darin, dass sich der Analytiker am Platz des Hörers befindet. Deuten in dem hier vorgestellten Zusammenhang basiert demzufolge auf dem Hören des Analytikers, dessen Aufmerksamkeit sich auf das Sprechen des Analysanden richtet. Als Hörer ist es an ihm, den Äußerungen seines Gegenübers einen (bestimmten) Sinn, also eine Bedeutung zu unterstellen.

Im Gegensatz zur alltagspraktischen Weltbewältigung, die in wachsendem Maße von kausalen Erklärungsmodellen auf empirischer Grundlage bestimmt ist, beruhen soziale Regelungssysteme im Wesentlichen auf Sinndeutungen. Diese Sinndeutungen sind sowohl Folge als auch Quelle für die Art und Weise, wie die Dinge und die zwischenmenschlichen Beziehungen ihre Ordnung haben.

Auf dieser Basis einer sog. symbolischen Ordnung legitimieren sich schließlich soziale Umgangsregeln, ethische Maßgaben, Sanktionen bei Verstößen etc. Im Prinzip handelt es sich bei geschriebenen und ungeschriebenen Gesetzen um relativ willkürliche Regelungen, die das Ergebnis existierender sozialer Machtverhältnisse und Traditionen sind.

Die Regeln und Gesetze, welche die symbolische Ordnung repräsentieren, die Grammatik der Sprache und die Wahrnehmung begründen ein Ergänzungsverhältnis, was weitreichende Konsequenzen für das reflexive Denken hat. Kurz gesagt: Die Sprache determiniert, leitet und begrenzt das Denken und den Wahrnehmungsbereich. Statt vieler, hier nur ein Beispiel zur Illustration, um das Problem zu verdeutlichen: Guy Deutscher zeigt in seiner Untersuchung, dass Sprachen, in denen unbelebte Objekte mit „er“ oder „sie“ behandelt werden, zur Konsequenz haben, „dass den Sprechern jedes Mal eine Assoziation zwischen einem unbelebten Substantiv und einem der Geschlechter in die Ohren geschoben wird, wenn sie den Namen 
dieses Objekts hören“ (2010, S. 238). Das mag auf den ersten Blick marginal erscheinen. Deutscher demonstriert aber die impliziten Folgen am Beispiel der Übersetzung des Gedichtes von Heine „Ein Fichtenbaum steht einsam“ ins Englische:

\begin{tabular}{ll}
\hline Ein Fichtenbaum steht einsam & $A$ pine-tree standeth lonely \\
Im Norden auf kahler Höh', & In the North on an upland bare; \\
Ihn schläfert; mit weißer Decke & It standeth whitely shrouded \\
Umhüllen ihn Eis und Schnee. & With snow, and sleepeth there. \\
$E r$ träumt von einer Palme, & It dreameth of a Palm Tree \\
Die, fern im Morgenland, & Which far in the East alone \\
Einsam und schweigend trauert & In mournful silence standeth \\
Auf brennender Felsenwand. & On its ridge of burning stone. \\
(Heinrich Heine) & (Übersetzung: James Thomson) \\
\hline
\end{tabular}

Da das Englische keine klare Unterscheidung des sprachlichen Geschlechts der Substantive macht, kann die Pointe nicht zünden, die in einer Allegorie besteht und dem englischen Leser vorenthält, dass es sich um ein Liebesgedicht und nicht um eine botanische Beschreibung handelt. Erst der Übersetzung von Emma Lazarus gelingt es, diese Anspielung auch im Englischen hörbar zu machen, weil sie anstelle des Neutrums ,it“ die für die deutsche Fassung passenden Geschlechtspronomina „he“ bzw. „her" einsetzt. Georges-Arthur Goldschmidt (1999, 2006) hat die jeweiligen Besonderheiten des Deutschen und des Französischen miteinander verglichen. Bettelheim (1988) hat dieses Sprachproblem luzide am Beispiel der Übersetzung Freudscher Texte ins Englische dargestellt.

Bevor nach diesen kursorischen Abhandlungen dieses Kapitel beschlossen wird, möchte ich darauf aufmerksam machen, dass in den nun folgenden Ausführungen immer dann, wenn von Sprache die Rede ist, die erwähnte symbolische Ordnung gemeint ist. In verbalen Äußerungen präsentiert sie sich in der dem Sprechen zugrundeliegenden Grammatik, in averbalen in den sozialen Konventionen und Gesetzen. Darüber hinaus sind die Begriffe symbolisch und Symbol nicht gleichzusetzen mit dem alltagssprachlichen Begriff des Sinnbildlichen, sondern meinen hier, dass unter Verwendung von Lauten und/oder Zeichen zwischenmenschliche Beziehungen artikuliert und geregelt werden.

Über die Frage, wie der Mensch als Gattungswesen zur Sprache gekommen ist, kann man nur spekulieren (Falk u. Kuhlmann-Krieg 2010). Anders sieht es dagegen aus bei der Frage, wie das Individuum, entsprechende anatomische Fähigkeiten vorausgesetzt, zur Sprache kommt. Spätestens mit der Geburt taucht das Kind in eine bereits bestehende Welt der Sprache ein und begegnet in den Handlungsvollzügen womit die Basiserfahrungen des prozeduralen Gedächtnisses gemeint sind - besagter symbolischer Ordnung, in der ihm pränatal von der Mutter bzw. den Eltern ein Platz zugewiesen worden ist, in dem zum Ausdruck kommt, welche Bedeutung das Kind im Leben der Eltern hat und haben wird. Denkt man beispielsweise an den Zeugungsakt: Ist das Kind ein Ergebnis von Liebe oder Gewalt? Wurde es gewünscht, ersehnt oder war es ungewollt? Welche Erwartungen verbanden und verbinden sich mit dem Kind?

Schließlich ist es die Namensgebung, die exemplarisch für den Prozess der Subjektwerdung des Kindes steht und im Taufakt den Körper des Kindes mit einbezieht. 
Von diesem Zeitpunkt an wird das Kind mit dem ihm zugedachten Namen gerufen und angesprochen. Gleichzeitig vermerken staatliche und kirchliche Stellen seinen Namen in ihren Büchern. Und es wird der Name sein, der nach dem Tod des Subjekts bleiben und in der symbolischen Ordnung von seiner ehemals leibhaftigen Existenz Zeugnis ablegen wird.

Im Sozialisationsprozess, der das Kind zur Sprache und zu seiner Identität bringt, spielen also Zuschreibungen, die als „Identifikationen durch jemanden“ (Knellessen et al. 2003) analytisch gefasst werden können, eine entscheidende Rolle. Sie werden, wie gesagt, an dem noch unbeschriebenen Teil der biologischen Ausstattung des Kindes vollzogen. Schritt für Schritt erwirbt das Kind mit dem Sprechen nicht nur seine Muttersprache, sondern auch eine darin enthaltene ganz typische Sicht auf die Welt und sich selbst - typisch im Hinblick auf das Land seiner Geburt und typisch im Hinblick auf die Familie, in der es aufwächst. Insofern ist das Subjekt selbst ein Produkt seines Sprechens, denn es hat nicht gelernt, seine Bedürfnisse mithilfe des Anderen in dessen Sprache auszudrücken - analog jemandem, der sich eine Fremdsprache aneignet, um sich verständlich zu machen. Vielmehr lernt es über die Zuschreibungen von anderen (bspw. der Mutter), welche Bedürfnisse es überhaupt hat bzw. zu haben hat. Da sogar Affekte und Gefühle, sobald sie als solche inhaltlich benannt werden, unwiderruflich Teil der symbolischen Ordnung werden, können sie nicht als Referenzpunkte für ein Außerhalb der Sprache herhalten, von wo aus man richtige von falschen Interpretationen des kindlichen Ausdrucks unterscheiden könnte. Selbstverständlich schränken die vitalen Grundbedürfnisse nach Nahrung, Wärme und Schutz den Interpretationsspielraum ein. Zumal sie und die Möglichkeiten ihrer Befriedigung in einem gewissen Umfang bei Mutter und Kind instinktiv verankert sind. Allerdings gibt es keine 100\%ige Sicherheit, dass die Erwachsenen auf die Signale des Kindes immer vorhersehbar reagieren; dies ist an solchen Extremen wie Kindstötung und -verstoßung zu belegen. Die menschliche Verfasstheit ist eben überwiegend ein Produkt der symbolischen Ordnung.

Es sind also diese Zuschreibungen, die in der Verknüpfung von ursprünglichen vegetativen Reiz-Reaktions-Schemata mit Sprech- und Handlungsakten, den Nukleus des Subjekt des Bewussten, sprich: des Ich des Kindes legen. Zur Sprache kommen bedeutet also auch, allmählich und unwiderruflich aus dem ursprünglichen narzisstischen Welterleben, aus der mütterlichen Symbiose herauszufallen. Und je mehr der Mensch die Sprache bewohnt, zu einem Sprechwesen wird, desto mehr wächst der Abstand zu seinem vegetativen Sein. Insofern ist der Preis der Subjektwerdung oder Individuation buchstäblich eine Vereinzelung, die dem Subjekt in Form einer basalen Ich-Spaltung als Mal eingeprägt und als ontologische Selbstentfremdung Grundlage der Conditio humana ist.

Weil die Mutter im Schrei des Babys dessen Anspruch auf Bedürfnisbefriedigung zu vernehmen meint und das unterstellte Bedürfnis stillt, transformiert sich der Schrei allmählich für beide zu einem Ruf, zu einem Appell. Man kann darin den Kristallisationskern für weitere Lautproduktionen entdecken, aus denen das Kind nach und nach Worte und später ganze Sätze formen wird.

Schließlich verfügt das Kind mit dem Sprachvermögen über ein mentales Reflexionsmedium, mit dessen Hilfe es nicht nur die abwesende Mutter, sondern auch andere Objekte, denken und imaginieren kann. Es wird damit für das Kind zu einem Trostspender im Kummer der Trennung, weil es das abwesende begehrte Objekt im 
Laut symbolisch anwesend sein lassen kann. Letztlich sind es die unvermeidlichen Erfahrungen von Mangel und Abwesenheit, die das Kind dazu nötigen, die basalen intrapsychischen Strukturen zu entwickeln, die letztendlich konstitutiv für das IchDu-Bewusstsein ist. Subjektwerdung und Sprechenlernen sind also nur zwei Seiten ein und derselben Medaille: Nur weil Worte auf etwas verweisen, das sie selber nicht sind, bedeuten sie dem Subjekt etwas.

\section{Von der Psychoanalyse}

Unter Verweis auf das Phänomen der Regression wird die psychoanalytische Beziehung gern mit der frühen Abhängigkeitsbeziehung des Kindes von seiner Mutter bzw. seinen Eltern verglichen. Eine solche Sichtweise lokalisiert demzufolge den Analysanden wie selbstverständlich auf dem Platz des Rat- und Hilflosen. Demgemäß entspringt seine Übertragungsbereitschaft seiner Annahme, der Analytiker verfüge über das Wissen, was ihm fehlt.

Komplementär dazu findet sich auf Seiten des Analytikers die Überzeugung als Angehöriger einer Profession von Deutern, über eben das unterstellte Wissen und die Befähigung zu verfügen, Fremdseelisches zu verstehen.

Dabei ist jedoch zu bedenken, dass der Analysand trotz der unterstellten regressiven Tendenzen ein Erwachsener bleibt. Freud unterscheidet nicht von ungefähr zwischen der Nacherziehung eines erwachsenen Neurotikers und der Erziehung eines Kindes, denn ein Kind sei ,eben noch kein Neurotiker und Nacherziehung etwas ganz anderes als Erziehung des Unfertigen“ (1925f, S. 566). Der zwischen einem erwachsenen Neurotiker und einem Kind bestehende Unterschied liegt im Wesentlichen darin, dass er über eine ausgebildete Sprachkompetenz verfügt, die ihn im Allgemeinen relativ stabil in der symbolischen Ordnung verankert. Eine Ausnahme stellen Erwachsene dar, die eine psychotische Struktur besitzen und deswegen den Prozess der Alienation nur unzureichend durchlebt haben, so dass es in Krisensituationen leicht dazu kommt, dass sie Worte wie Objekte erleben.

Zwar tritt der Patient - darin gleicht er dem präverbalen Säugling - dem Analytiker als appellierendes Subjekt gegenüber, das eine Befriedigung seiner vorgetragenen Bedürfnisse erwartet. Gleichzeitig artikuliert er aber in seinen Symptomen - als Folge der bereits erwähnten Selbstentfremdung - die Frage nach seinem Sein; eine Frage, die darum kreist, wer er eigentlich ist und die sich nun strukturell an den Analytiker am Platz des Wissenden adressiert.

Indem man am Beginn einer Analyse diese Unterstellung des Patienten zunächst unkommentiert akzeptiert, stützt man eine Variante idealisierender Übertragung, die jedoch für das Zustandekommen eines analytischen Diskurses unverzichtbar ist, weil „erst durch diese Illusion eines vorgängigen Wissens (...) am Ende das wirkliche Wissen hervorgebracht werden“"kann (Žižek 1988, S. 168).

\section{Das wirkliche Wissen}

Zu dem wirklichen Wissen kann der Patient im Verlauf der Analyse letztlich nur selber finden, weil es in ihm begründet liegt, auch wenn er anfangs noch davon überzeugt 
ist, den Sinn seiner Symptome nicht zu kennen (Freud 1916-17a, S. 98). Etabliert sich nach und nach ein analytischer Diskurs, wandelt sich der Patient vom Leidenden zum Fragenden und setzt auf diese Weise einen Prozess des Sich-Selbst-Verstehens in Gang, an dessen Ende er neben vielem anderen auch die Antwort auf seine existenzielle Frage findet, die da lautet, dass es keine end-gültige Antwort gibt außer der, die er sich selber gibt.

An diesen Punkt kann ein Analysand jedoch nur gelangen, wenn sich auch der Analytiker darüber im Klaren ist, selbst ein gespaltenes Subjekt zu sein - ein Subjekt, aus dem es spricht, mag er sich auch noch so sehr an die Illusion klammern, selbst zu sprechen (Borens 1988). Ein Festhalten an dieser Illusion aber lässt den analytischen Selbstfindungsprozess scheitern und verwandelt die strenge tendenzlose Analyse, wie sie Freud (1919a) ursprünglich vorschwebte, in ein psychagogisches Vorgehen. Sobald Analytiker meinen, dem Patienten ein Wissen über sich vermitteln zu müssen, vermitteln sie in Wahrheit nur ihr eigenes Weltbild. Und das geschieht, weil dabei übersehen wird, dass man sich nur am Platz des Wissenden befindet und nicht selbst der Wissende ist.

Aus struktural-analytischer Sicht wird der Platz des Wissenden auch bezeichnet als der Ort der Sprache, die Trägerin des Unbewussten ist, das daher - wie oben skizziert - unabhängig vom konkreten Individuum und daher außerhalb seiner selbst existiert, wenngleich es über die Sprache auf es einwirkt.

Im Falle eines solchen strukturellen Missverständnisses, das Platz und Person als eins setzt, werden durch die gegebenen Deutungen nicht die Suche des Patienten nach seiner Frage und seiner Antwort, die in seinem Symptom verschlüsselt liegen unterstützt, sondern in erster Linie die latenten narzisstischen Bedürfnisse des Analytikers nach Anerkennung befriedigt, der nolens volens den Analysanden dahingehend manipuliert, sich mit den gegebenen Deutungen bzw. Zuschreibungen zu identifizieren: „Genau das aber sollte die Position des Analytikers nicht sein, er sollte der (symbolische) Andere sein, der immer kastriert, unvollständig ist, nicht wissend und schon gar nicht allwissend“ (Borens 2013, S. 177).

\section{Exkurs}

Im Sommer 1901 fand ein letztes Treffen zwischen Freud und Fließ am Thumsee statt. Offenbar war dort eine wachsende tiefgreifende Entfremdung zwischen beiden nicht mehr zu übersehen gewesen, was im Nachgang schließlich den endgültigen Bruch der Beziehung eingeleitet hatte. Zumindest findet sich in einem unmittelbar nach diesem Treffen von Freud geschriebenen Brief an Fließ folgende Textpassage: „Und auch Du bist hier an die Grenze Deiner Scharfsichtigkeit gekommen, nimmst Partei gegen mich und sagst mir, was alle meine Bemühungen entwertet: Der Gedankenleser liest bei den anderen nur seine eigenen Gedanken. Wenn ich so einer bin, so wirf mein Alltagsleben nur ungelesen in den Papierkorb“ (Freud 1985c, S. 492). Leider fehlen die Briefe von Fließ, die mehr Einblick hätten geben können, was dort eigentlich abgehandelt worden war. 
Wenigstens so viel kann indes aus Freuds heftiger Reaktion geschlossen werden, nämlich dass ihm das erkenntnistheoretische Dilemma psychoanalytischer Deutungen und eines eindeutigen wissenschaftlichen Beweises für ihre Richtigkeit schwante.

Wissenschaftsgeschichtlich betrachtet ist das hypnotische Verfahren die grundlegende Matrix der psychoanalytischen Theorie und Praxis (Mayer 2002). Obwohl Freud (1916-17a) die mäeutische Funktion der Hypnose für die Entstehungsgeschichte der Psychoanalyse niemals geleugnet hat, sah er in ihr später dennoch eine Art Schmuddelkind, von dem sich die nachfolgenden Analytikergenerationen besser fernzuhalten hätten. Auf diese Weise hoffte er, den stets virulenten Vorwurf zu entkräften, die Psychoanalyse sei wie die Hypnose nur eine verkappte Form suggestiver Einflussnahme.

Anfangs bestand das psychoanalytische Vorgehen zwar im Erraten des unbewussten Sinns neurotischer Symptome unter Zuhilfenahme der Einfälle des Analysanden. Wegen der nach und nach aufkommenden Störungen im Assoziationsfluss seiner Patienten, sah sich Freud jedoch bald zu einer Modifikation seines Verfahrens gezwungen. Immer dann, wenn der Patient nunmehr behauptete, keine Einfälle mehr zu haben, legte er ihm seine Hand auf die Stirn, um damit einen sanften Druck auszuüben, begleitet vom Hinweis, der Patient möge danach sagen, welcher Gedanke ihm dabei plötzlich durch den Kopf gegangen sei.

Freud schreibt weiter, es sei ihm so vorgekommen, als habe er mit dem Druck seiner Hand eine ,psychische Kraft bei dem Patienten [überwunden], die sich dem Bewusstwerden (Erinnern) der pathogenen Vorstellungen (Freud 1895d, S. 268) widersetzt habe. Dieser Analogieschluss brachte die bis heute gültige Verdrängungsund Widerstandstheorie der Psychoanalyse hervor.

Im Konzept der Übertragungsanalyse (Freud 1912b, S. 366) stand diese Idee noch einmal Pate. Weil Freud glaubte beobachtet zu haben, dass die Ursache vieler Lücken im Fluss der freien Einfälle mit einer Verschiebung, sprich: Übertragung des Patienten auf seine Person zusammenhing, griff er zu einer quasi virtuellen Variante des Handauflegens, indem er dem Analysanden sagte, er sei wohl gerade in Gedanken mit ihm beschäftigt. Indem Freud die „Stockung“ darüber hinaus als Widerstand begriff - genauer: als Übertragungswiderstand -, den es zu überwinden galt, ließ er unhinterfragt, warum er meinte, immer in die ,Lücken“ des Assoziationsflusses seiner Analysanden einspringen zu müssen. Möglich, dass darin ein Hinweis enthalten ist, welche Nöte des Analytikers sich hinter gewissen Ratschlägen zur Behandlungstechnik verstecken.

Widerstands- und Übertragungsanalyse sind inzwischen die Kernstücke einer Behandlungstechnik, auf die Psychoanalytiker hinweisen, wenn sie ihr Vorgehen von einem psychotherapeutischen Verfahren abgrenzen wollen.

Gleichzeitig sind Widerstands- und Übertragungsanalyse aber auch die Achillesferse psychoanalytischer Technik schlechthin, weil sie im Zentrum des methodischen Problems stehen, auf welche Weise Deutungen, oder präziser gesagt, analytische Konstruktionen (Freud 1937d) verifiziert werden können. Gewöhnlich hält man eine Deutung für zutreffend, wenn sie: a) der Betroffene selbst bestätigt hat; b) das Symptom beseitigt oder gelindert wurde; c) weiterführende Einfälle auftauchen. Trifft keines dieser Prüfkriterien zu, muss das noch nicht heißen, dass eine gegebene Deutung oder Konstruktion falsch gewesen ist. In einem solchen Fall griff Freud auf das 
Prinzip der Exhaustion zurück, indem er Zusatzbedingungen einführte, die es ihm ermöglichten, seine Hypothese weiterhin beibehalten zu können.

Argumentativ versuchte er seine Ansicht durch den Vergleich der analytischen Situation mit einer Gerichtsverhandlung zu untermauern (Freud 1916-17a). Bei einer Gerichtsverhandlung ginge es so zu, dass der Richter bei Vorliegen eines Geständnisses die Richtigkeit der Beschuldigung annehmen und den Angeklagten verurteilen würde. Das Fehlen eines Geständnisses würde umgekehrt aber nicht automatisch zum Freispruch des Angeklagten führen. Vielmehr würde dann das Verfahren mit Hilfe von Indizien solange weitergeführt, bis sich der Richter in der Lage sieht, eine endgültige Entscheidung zu treffen. Nicht anders würde man in der Analyse verfahren, wenn sich Widerstände gegen psychoanalytische Deutungen oder Konstruktionen manifestierten. Man würde dann an der Richtigkeit einer Deutung festhalten, sofern ausreichend überzeugende Indizien vorliegen, denen schließlich die gleiche Dignität zukäme, wie den Wahrscheinlichkeitsaussagen empirischer Wissenschaften.

\section{Die Deutungsgewissheit Freuds}

Freuds Deutungsgewissheit erklärt sich zum einen aus seinen klinischen Lehrjahren mit der Hypnose und der aus ihr hervorgegangenen Dechiffrier-Methode, die ihn zu der Überzeugung gebracht hatte: ,Je mehr man dergleichen Rätsel bereits gelöst hat, desto leichter wird man vielleicht ein neues erraten“ (Freud 1895d, S. 285 f.). Zum anderen erklärt sie sich aus seiner Hypothese von der Reziprozität menschlichen Seelenlebens: „Ein beständiger Strom von „Eigenbeziehung“ geht so durch mein Denken, von dem ich für gewöhnlich keine Kunde erhalte. (...) Es ist, als wäre ich genötigt, alles, was ich über fremde Personen höre, mit der eigenen Person zu vergleichen, als ob meine persönlichen Komplexe bei jeder Kenntnisnahme von anderen rege würden. Dies kann unmöglich eine individuelle Eigenheit meiner sein; es muss vielmehr einen Hinweis auf die Art, wie wir überhaupt „Anderes“ verstehen, enthalten. Ich habe Gründe anzunehmen, dass es bei anderen Individuen ganz ähnlich zugeht wie bei mir" (Freud 1901a, S. 30 ff.).

In der Psychopathologie des Alltagslebens findet sich eine kleine Geschichte, die diese Überlegungen Freuds gut illustriert. Darin geht es um zwei allein reisende Männer, die sich in einem Hotel begegnen, sich anfreunden und mehrere Tage mit gemeinsamen Wanderungen miteinander verbringen. Morgens und abends sitzen sie bei den Mahlzeiten am selben Tisch des Hotels.

Plötzlich ändert sich das Verhalten des einen, der am nächsten Tag die Ankunft seiner Frau erwartete. Der andere hat das Gefühl zu stören und zieht sich daraufhin zurück. Als er tags darauf einer Einladung zu einem Essen zu dritt folgen will, muss er bei seinem Eintreffen feststellen, dass auf dem noch einzigen freien Platz am Tisch der Mantel des anderen liegt, und er versteht ,, sehr wohl den Sinn dieser gewiss nicht absichtlichen, aber darum umso ausdrucksvolleren Lagerung. Es hieß: Für dich ist hier kein Platz, du bist jetzt überflüssig“ (Freud 1901a, S. 235). Der andere merkt nicht, wie er vor dem Tisch stehen bleibt, ohne sich zu setzen, ,wohl aber die Dame, die ihren Mann sofort [anstößt und ihm zuflüstert]: Du hast ja dem Herrn den Platz verlegt" (Freud 1901a). 
Freuds Fazit aus dieser kleinen Vignette lautet: „Bei diesem wie bei anderen ähnlichen Ergebnissen habe ich mir gesagt, dass die unabsichtlich ausgeführten Handlungen unvermeidlich zur Quelle von Missverständnissen im menschlichen Verkehr werden müssen. Der Täter [sic!], der von einer mit ihnen verknüpften Absicht nichts weiß, rechnet sich dieselben nicht an und hält sich nicht verantwortlich für sie. Der andere hingegen erkennt, indem er regelmäßig auch solche Handlungen seines Partners zu Schlüssen über dessen Absichten und Gesinnungen verwertet, mehr von den psychischen Vorgängen des Fremden, als dieser selbst zuzugeben bereit ist und mitgeteilt zu haben glaubt. (...) Genau besehen beruht ein solches Missverständnis auf einem Zufein- und Zuvielverstehen. Je „nervöser“ zwei Menschen sind, desto eher werden sie einander Anlass zu Entzweiungen bieten, deren Begründung jeder für seine eigene Person ebenso bestimmt leugnet, wie er sie für die Person des anderen gesichert annimmt“ (Freud 1901a).

Prämissen und Schlussfolgerungen dieser Überlegungen haben die Grundsteine für eine analytische Behandlungstechnik gelegt, in der dem Patienten die Rolle eines Täters zugeschrieben wird, der die wahren Motive seines Handelns nicht kennt und von ihnen nichts weiß, während der Analytiker die Rolle eines Richters einnimmt, der sein Gegenüber beobachtend durchschaut und dessen Handlungen interpretiert, indem er im Analogschluss auf sich selbst bestimmte unbewusste Motive unterstellt. Die Hypothese allgemeiner Reziprozität im menschlichen Seelenleben kann ihre Legitimität letztlich nur aus den jeweils gültigen gesellschaftlichen Konventionen beziehen, wenn man nicht biologistische Anleihen machen will. Im Übrigen bliebe nur noch die Alternative, die subjektive Weltsicht des Deuters selbst zum Standardmaß der Korrektheit einer Interpretation von Fremdseelischem zu nehmen. Um diesem erkenntnistheoretischen Dilemma zu entkommen, das sich aus der Annahme der Reziprozität im menschlichen Seelenleben ergibt, schlägt Borens (1993) vor, auf der Ebene der Worte selbst zu bleiben und zu hören, statt zu versuchen, aus dem vom Analysanden Gesagten mit Hilfe der eigenen Gegenübertragungsreaktionen etwas Verborgenes zu destillieren.

Die von Freud erwähnte Gefahr von Missverständnissen ist im Allgemeinen dann besonders gegeben, wenn eine Interpretation vom Interpretierten als Unterstellung erlebt wird, so dass dieser sich tatsächlich zum Täter gestempelt fühlt. Dabei sind im analytischen Kontext nicht nur die Konsequenzen tragischer Entzweiungen relevant, die in letzter Konsequenz zum Abbruch einer Analyse führen würden, sondern vielmehr die latente Etablierung eines sog. Herrendiskurses (Lacan 1986, S. 21), der einem grenzüberschreitenden Verhalten des Analytikers Vorschub leistet. Das gilt insbesondere für die Fälle, in denen dem Analytiker die Macht gegeben ist, seine Sicht der Dinge unter offener oder versteckter Androhung bzw. Anwendung institutioneller Gewalt durchzusetzen.

\section{Die Einführung der Lehranalyse}

Mit der Einführung einer obligaten Lehranalyse war auch die Hoffnung verknüpft, dem wilden Analysieren, das ohne ausreichende Berücksichtigung der Situation des Patienten vor sich geht, einen Riegel vorzuschieben. Interessanterweise war Freud 
weniger darum besorgt, welchen Schaden das beim Patienten anrichten könnte, als mehr darum, welchen Schaden es der Sache der Psychoanalyse zufügen würde (Freud 1910k, S. 125). Inzwischen kann man ernüchtert feststellen, dass die Lehranalyse letztlich nicht geeignet war und ist, das Phantasma der Deutungsgewissheit aufzulösen. Womöglich hat sie in manchen Fällen diesem Phantasma sogar weiter heimlich Nahrung geben können. Paula Heimann problematisierte zwar schon 1950 genau dieses von der Gesamtgruppe der Psychoanalytiker latent geteilte Phantasma, mit dem Abschluss der Lehranalyse purgiert - um einen Ausdruck Freuds zu gebrauchen - und gegen Deutungsfehler relativ immun zu sein. Tatsächlich hat aber die von Heimann vollzogene Neubewertung der Bedeutung und Funktion der Gegenübertragung für den analytischen Prozess jedoch nur oder immerhin - je nach Standpunkt - erreicht, dass dieses Phantasma benannt wurde. Kläui (2002) sieht eine enge Verknüpfung zwischen der Frage der Gegenübertragung und der persönlichen Motivation, den Beruf des Analytikers zu wählen. Er hebt zwei Aspekte hervor, die maßgeblich an der Ausgestaltung der Gegenübertragung beteiligt sind:

1. der Wunsch, sich als Psychoanalytiker bezeichnen zu dürfen und

2. das Vermögen, als Psychoanalytiker arbeiten zu können.

Der Wunsch, Analytiker sein zu wollen, durchkreuzt als Gegenübertragung ganz entscheidend die gleichschwebende Aufmerksamkeit. Heimann geht deswegen mit ihren Schlussfolgerungen - im Wesentlichen basierend auf ihren Supervisonserfahrungen mit Ausbildungskandidaten - in die Irre, wenn sie die Probleme in der therapeutischen Dyade verortet, weil sie in ihren Überlegungen die triadische Struktur der Supervisionsbeziehung außer Acht gelassen hat. Wird diese mitberücksichtigt, so Kläui, dann zeigt sich, dass die Ausbildungskandidaten von dem Wunsch getrieben waren, von der Supervisorin als Analytiker gesehen zu werden. Statt einer psychischen Reziprozität oder Symmetrie das Wort zu reden, spricht sich Kläui dafür aus, das Fremde zur Grundlage der analytischen Begegnung zu machen und ,einfach zuzuhören (...) und (...) das Nichtverstehen auszuhalten“ (Kläui 2002, S. 64). Dass dieser Wunsch nicht nur ein Kennzeichen analytischer Novizen ist, sondern auch erfahrene Analytiker umtreibt, habe ich an anderer Stelle ausgeführt (Klemann 2008).

Solange man als Analytiker noch vom Begehren, der Wissende sein zu wollen geleitet wird, hat man das eigene Begrenztsein nicht akzeptiert - Lacan verwendet dafür den Passus des Annehmens der Kastration - und sorgt nolens volens dafür, dass sich das Unbewusste in der Analyse mehr und mehr verflüchtigt (Bollas 1997, 2006, Pohlen u. Bautz-Holzherr 1991); vorausgesetzt, man definiert das Unbewusste als das, was man nicht weiß, also auch nicht wissen kann. Die scheinbare Gewissheit, die unbewusste Dynamik und Bedeutung eines Symptoms des Patienten zu verstehen, weil man über das entsprechende professionelle Wissen und Können zu verfügen glaubt, führt auf direktem Wege zu der Überzeugung, dass der Patient sich im Widerstand befindet, wenn er eine Deutung ablehnt. Die Bevorzugung der Widerstandsanalyse in der Behandlungstechnik ist darum die logische Konsequenz einer solchen Haltung.

Eine Variante der Widerstandsanalyse ist die Übertragungsanalyse. Während Freud die Übertragung ursprünglich als ein lästiges Widerstandsphänomen kennen und später die sog. Übertragungsneurose als unvermeidbares Durchgangsphänomen 
begriff und zu nutzen gelernt hatte, um den Patienten von der Richtigkeit seiner psychoanalytischen Konstruktionen zu überzeugen, zielt die zeitgenössische Behandlungstechnik regelrecht darauf ab, durch Übertragungsdeutungen im Hier und Jetzt, möglichst rasch eine Übertragungsneurose herzustellen, in der Überzeugung, nur so das Heilungspotenzial der Psychoanalyse wirksam zur Geltung bringen zu können. König (2007) vermutet zudem als weiteren Grund für die Abneigung, sich mit den Außenbeziehungen des Patienten zu beschäftigen, dass solche Beziehungen zu unübersichtlich und die Außenobjekte unbekannt seien. Wurde früher die Übertragung i. S. von ,Sie reden zwar von mir, aber eigentlich geht es um die Beziehung zu dem und dem" gedeutet, heißt es mittlerweile, "Sie reden zwar von dem und dem, aber eigentlich meinen Sie doch mich!". Mit dieser Umkehrung wird dem Patienten von Anfang an ein Widerstand gegen die Übertragung und damit gegen das Herzstück analytischer Heilungsbemühungen unterstellt.

Ein geradezu erschütterndes Lehrstück findet sich bei Thomä (2008). Eine Analysandin hatte sich brieflich an ihn gewendet, nachdem sie ihre Analyse abgebrochen hatte, weil ihr damaliger Analytiker ihre Einwände auf seine Deutungen notorisch als Angriffe auf die therapeutische Beziehung interpretiert hatte. Thomäs Ausführungen zeigen, dass der im Ergebnis destruktive Verlauf der Analyse eine wesentliche Ursache in der unreflektierten Position des Analytikers selbst hatte. Dieser hatte nämlich übersehen, dass er sich per Übertragung lediglich auf dem Platz des Wissens befand. Folglich hatte er ihre Einwände oder Kritiken primär als unbewusst motivierte Widerstandsreaktionen eingeschätzt. Tatsächlich verfügte er jedoch über das Wissen ebenso wenig wie die Analysandin. Damit steht dieser Analytiker in einer Tradition, die Widerstandsreaktionen bei Analysanden sowohl als die Indikatoren betrachtet, ein wirklicher Analytiker zu sein, als auch in ihnen den rettenden Ariadnefaden sieht, der im Labyrinth des analytischen Prozesses die notwendige Orientierung geben kann; signalisieren ihm die Widerstände doch, sich auf dem richtigen Weg zu befinden. Der Widerstand gegen die Übertragungsdeutung ist jedoch ein Artefakt derselben. Analog zum Sprachapriori, das besagt, dass es außerhalb der Sprache nichts gibt, worüber man sinnvolle Aussagen machen kann, gibt es außerhalb der analytischen Situation, sprich: der Übertragungsbeziehung, keinen Ort, von dem her die Übertragung als solche gedeutet werden könnte. Die Übertragung kann daher nur innerhalb des Mediums der Sprache als signifikantes Ereignis im Diskurs - sprich: als etwas, das sich im Sprechen zeigt - markiert, aber nicht gedeutet werden.

Jede Deutung mobilisiert automatisch Widerstand, weil sie von der Person kommt, „die zu sein ihm die Übertragung auferlegt“ (Lacan 1996, S. 179), wodurch alles, was dazu gesagt wird durch die Übertragung kontaminiert ist. Da ein Analytiker letztlich auch nicht mehr ist als ein Mensch wie jeder andere, siedelt jede Deutung auf der IchEbene, die im Glauben an eine gemeinsame Realität gründet und unhinterfragt voraussetzt, dass der gesunde Teil des Ich des Patienten genauso denkt wie man selbst. Ein Klassiker dieser Annahme ist bekanntlich das Konzept der therapeutischen IchSpaltung (Sterba 1934).

Manchen Analytikern galt und gilt es sogar außerhalb des analytischen Settings irrigerweise als Beweis im Besitz einer wahren Erkenntnis zu sein, sobald sie mit ihren Ansichten auf Ablehnung oder Kritik stoßen. Bedauerlicherweise kann sich eine solche Überzeugung auch noch auf eine Tradition berufen, die Freud mit seiner 
Aussage begründet hatte, dass es der Psychoanalyse gewissermaßen systemimmanent sei, Ablehnung zu provozieren, weil ihre Einsichten die Menschen zum Widerspruch reizen und erbittern muss (Freud 1914d, 1940a).

\section{Zur Praxis des Deutens und des Selbstverständnisses des Analytikers}

Wenngleich Freud die Arbeit an und in der Übertragung stets als Mittel zum Zweck der Konstruktion einer verdrängten und traumatisch verarbeiteten Lebenserfahrung des Subjekts gesehen hat (Freud 1937d), haben sich in seiner Nachfolge mittlerweile unterschiedliche Vorgehensweisen in der Durchführung einer Analyse etabliert, in denen dem Deuten ganz spezifische und verschiedene Funktionen zukommt. Hier sind kontrapunktisch zwei Richtungen zu nennen, von denen die eine in der Tradition der Nacherziehung und die andere in der Tradition des Findens steht.

Die allmähliche Verschiebung des Fokus der Analyse auf die Übertragungsbeziehung war Freuds Tribut an die sog. therapeutische Aufgabe, womit er die Nacherziehung stärker ins Zentrum der Behandlungstechnik rückte. Im Kern ging es ihm darum, ,den Kranken dazu zu bringen, daß er aus Motiven besserer Einsicht etwas akzeptiert, was er zufolge der automatischen Unlustregulierung bisher zurückgewiesen (verdrängt) hat" (Freud 1905a, S. 25; s. a. 1914d, S. 448 ff.). Noch deutlicher zeigt sich diese quasi pädagogische Ausrichtung der Analyse in der Beschreibung der behandlungstechnischen Funktion der Übertragung: „In der Psychoanalyse arbeiten wir an der Übertragung selbst, lösen auf, was ihr entgegensteht, richten uns das Instrument zu, mit dem wir einwirken wollen“ (Freud 1916-17a, S. 469). Damit wurde die Übertragung des Patienten zum archimedischen Punkt, von dem aus sich der Analytiker nunmehr berechtigt fühlt, die Suggestion zum Wohl des Patienten als Hebel einsetzen zu dürfen: „Nicht der Kranke suggeriert sich allein, wie es in seinem Belieben steht, sondern wir lenken seine Suggestion, soweit er ihrem Einfluss überhaupt zugänglich ist" (Freud 1916-17a, S. 470).

Im Klartext wird damit der Einsatz kontrollierter, vom Analytiker gesteuerter Suggestionen in der Analyse gerechtfertigt, weil das die sog. therapeutische Aufgabe verlangt. Erst recht galt dies nach Freud für die Massenanwendung der Analyse, die es notwendig macht, ,das reine Gold der Analyse“ - womit deren „Strenge“ und „Tendenzlosigkeit“" gemeint war - ,reichlich mit dem Kupfer der direkten Suggestion zu legieren“" (Freud 1919a, S. 193).

Gegenwärtig begegnet man unter dem Etikett der Britischen Schule einer Variante der analytischen Behandlungstechnik, die - wie ich es nennen möchte - der forcierten „Übertragungsanalyse im Hier und Jetzt“ den Vorzug gibt. Vertreter dieser Richtung beanspruchen für sich zwar, die Bewahrer einer strengen und tendenzlosen Analyse zu sein, übersehen dabei aber leicht die suggestiven Elemente ihre Vorgehensweise. Bollas (2006) kritisiert an dieser Behandlungstechnik deren normative Ambitionen und einseitige Bevorzugung der Übertragungsanalyse. An die Stelle eines gemeinsamen Nichtwissenkönnens angesichts der Bildungen des Unbewussten (Symptome, Träume, Parapraxien) seien repetitive Vorgehensweisen und Deutungsfiguren getreten. Typisch dafür sei in kasuistisch-technischen Seminaren die notorische Frage: Und was ist mit der Übertragung? sowie die sog. Wochenend-Deutung. Letztere 
bezieht ihre Prominenz und Legitimation wohl zum einen aus Freuds Beschreibung der sog. ,Montagskruste“, und zum anderen aus verschiedenen Annahmen der Kleinschen Sadismustheorie sowie aus der Bindungstheorie. Auf jeden Fall wird in Analysen immer dann gerne die Wochenend-Deutung verwendet, wenn zeitnah um das Wochenende herum emotionale Reaktionen oder Phänomene beim Patienten auftauchen, die als Beleg für das Vorhandensein eines unbewussten Widerstandes gegen die vermutete reaktive Trennungsaggression betrachtet werden.

Borens zitiert zur Illustration einen typischen Fall, in dem eine Analytikerin den Ärger ihrer Patientin als verschobene Wut des kleinen Mädchens in ihr deutet, das ,, richtig Krach schlagen möchte, weil es sich herumgeschubst fühlt und mit Phantasien allein gelassen wird, [was die Therapeutin] während des Wochenendes wohl tue“ (Borens 1993, S. 36). Wenngleich die Analytikerin explizit betont, sie habe versucht, sich auf den für sie zentralen Bedeutungsgehalt des von der Patientin mitgeteilten Traumes zu konzentrieren, denn um einen solchen ging es in der Behandlungsvignette, übersieht sie die implizite suggestive Einflussnahme, indem sie die Aufmerksamkeit der Patientin von möglichen Einfällen zum Traum weg auf sich als Übertragungsobjekt zieht.

Eine vom Prinzip her gelegentlich zutreffende Beobachtung verkommt damit zu einer Deutungsfigur, womit die gleichschwebende Aufmerksamkeit der Analytikerin aufgehoben wird.

Deutungsroutinen von dieser Art sind beinahe die zwangsläufige Konsequenz des genannten Phantasmas, verstehend im Besitz des Wissens über das Unbewusste des Anderen zu sein. Ähnlich problematisch stellt sich der Einsatz der Gegenübertragungsanalyse dar, so weit man sinnvoll von deren Möglichkeit überhaupt sprechen kann. Besonders steht sie in Gefahr, esoterische Züge anzunehmen, wenn sie als unmittelbarer Zugang zum Unbewussten des Patienten begriffen wird. Anstatt zu hören, was der Patient sagt, richtet der Analytiker in einem Zustand von Rêverie sein Ohr auf seine Innenwelt und lauscht auf Signale, die er von dort herkommend vernimmt, im Glauben, so dem authentischen unbewussten Erleben des Patienten und der davon tangierten Beziehungsdynamik in der Analyse am nächsten zu sein. König (2010) sieht in der Rêverie die Gefahr, ,dass der Analytiker in diesem Zustand zu Überzeugungen gelangt, die er im fokussierenden Zustand nicht überprüfen und hinterfragen kann oder will“" (S. 169). Außerdem bleibt unreflektiert, dass sich dieses vermeintliche Verstehen der unbewussten Dynamik primär aus der Wahrnehmung der bewussten Affekte ableitet.

In den Pionierzeiten der Psychoanalyse hatte Freud seine Hauptaufgabe darin gesehen, den Fluss der freien Assoziationen möglichst von Störungen frei zu halten und aufkommende Hindernisse aus dem Weg zu räumen. In eben dieser Tradition steht eine Behandlungstechnik, die auftretende Stockungen in möglichst vieldeutiger Weise aufgreift und anspricht, um den Analysanden im positiven Sinne zu irritieren und ihn in die Lage zu versetzen, selber zu hören, was er unbewusst zur Sprache gebracht hatte. Das primäre Ziel dieser Deutungsaktivität entspringt der Absicht, die Vieldeutigkeit des Gesprochenen aufscheinen zu lassen und nicht, ein wie auch immer geartetes Wissen zu schaffen. Insoweit der Analytiker mit seinen Deutungen das Sprechen des Analysanden unterbricht, also buchstäblich interveniert, ermöglicht er es ihm, innezuhalten und sein eigenes unverstandenes Sprechen zu hören. Dafür ist 
es nicht nötig, dass der intervenierende Analytiker den Patienten bzw. dessen Unbewusstes versteht oder verstanden haben muss, womit die grundlegende Aporie des analytischen Diskurses aufscheint, nämlich wie sich diese Interventionen legitimieren und was ihr Spezifikum ist?

Borens (1988) antwortet auf die Frage nach dem Wesen einer Deutung, dass sie aus seiner Sicht stets „eine Mitteilung [ist], die der Analysand in seinem Sprechen über den Umweg des Analytikers sich selbst gibt" (S. 207). Damit betont Borens die Paradoxie jeder analytischen Beziehung, die darin besteht, dass nicht der Analytiker, sondern der Analysand, auch wenn dieser manifest vom Gegenteil überzeugt ist, über sich am besten Bescheid weiß.

Demnach geht es in der analytischen Praxis mitnichten um das Deuten von etwas Latentem, so verführerisch dieser Gedanke auch sein mag, sondern vielmehr um ein An-Deuten. Ziel dabei ist es, den Patienten anzuregen, seine unbewusste Frage nicht an den Analytiker zu richten, sondern sich selber - im wahrsten Sinne des Wortes - in Frage zu stellen, und ihm damit die Möglichkeit zu eröffnen, eine Ahnung davon zu bekommen, dass er selbst seine Frage ist; dass er nach sich selber fragt!

Bruce Fink (2005) lokalisiert im Auftauchen der Frage: Was ist mit mir los? ebenfalls den entscheidenden Wendepunkt der Analyse, den er als den Beginn des Subjektivierungsprozesses bezeichnet, der sich fundamental von einem Prozess der Nacherziehung unterscheidet. Das ist schließlich der entscheidende Punkt für Lacan, das Konzept des Verstehens zu problematisieren. Ein Verstehen, das auf der Anwendung des analytischen Wissenskanons und der Gegenübertragungsanalyse ruht, kann nur das Ergebnis einer verkappten und daher unerlaubte Einflussnahme auf den Analysanden hervorbringen. Nicht zuletzt deswegen warnte Lacan in seinen Kontrollanalysen: „Geben Sie vor allem acht, daß Sie den Kranken nicht verstehen, es gibt nichts, das Sie mehr irre macht als das" (Lacan, 1991, S. 115). Unter Berufung auf Paula Heimann (1954) würden viele glauben, dass das Verstehen unverzichtbar für einen erfolgreichen Verlauf der Analyse sei. Tatsächlich sei ein Konzept, das das Verstehen ins Zentrum der Analyse rücke nichts anderes als ein ins Professionelle gewendetes Symptom, dessen Funktion darin bestünde, die Angst abzuwehren, die angesichts des Fremden und Unverständlichen beim Analytiker entbunden wird: Man ,will die Angst nicht wahrhaben, die vom Andern (...) ausgeht dadurch, dass er nicht ein Ähnliches ist“" (Lacan 1996, S. 206). Es ist dieselbe Angst, die in der Analyse jeweils die Schwellen markiert, vor denen der Patient vorübergehend zurückscheut, bevor er weitere Fortschritte machen kann.

Macht man mit dieser Perspektive ernst, dann ist die analytische Begegnung im Kern genauso eine verfehlte Beziehung, wie alle übrigen Begegnungen, weil Analysand und Analytiker sowohl von der Annahme psychischer Reziprozität ausgehen, als auch glauben, dass dieselben Worte jeweils dasselbe meinen, nur weil sie beide dieselbe Sprache sprechen. In Wahrheit bewegen sie sich kommunizierend vorwiegend im Imaginären, das ihnen vorgaukelt, das Ganze zu sein, denn die Sprache hat ,noch eine andere, private Existenz als ein System von Wissen, das jeder einzelne Sprecher in seinem oder ihrem Geist verinnerlicht hat" (Deutscher 2010). Die Grenze zur wilden Analyse ist dabei schnell überschritten, wenn bereits aus wenigen Informationen Diagnosen wie Horoskope erstellt werden, um daraus weitreichende Deutungen abzuleiten. Ein im Übrigen in kasuistischen Seminaren häufig zu beob- 
achtendes Phänomen, wenn die Anwesenden anfangen sich mit ihren Interpretationen wetteifernd zu überbieten, um die Deutungshoheit zu erlangen.

Ähnlich problematisch sind Äußerungen, in denen man sich als Analytiker selbst zum Maßstab für die Realitätsadäquatheit oder -inadäquatheit des Handelns, Fühlens und Denkens des Patienten nimmt. Interventionen wie: „Wenn ich Ihnen so zuhöre, was Sie mir da über Ihre Beziehung zu Ihrem Partner erzählen, wundere ich mich, dass Sie so ruhig dabei sind. Eigentlich müsste Sie das doch wütend machen!“ inthronisieren den Analytiker als Repräsentanten einer Norm. Auf diese Weise wird dann nur das Verhalten der Eltern wiederholt, wie Freud schreibt, ,die die Unabhängigkeit des Kindes durch ihren Einfluss erdrückt hatten, und man ersetzt nur die frühere Abhängigkeit durch eine neuere" (Freud 1940a, S. 100).

Eine zurückhaltende Deutungstechnik steht in einem relativ großen Kontrast zur Technik der forcierten Übertragungsanalyse. Letztere sei die Folge eines professionellen Deutungszwanges: statt dass der Analytiker sich - wie Borens (1988) schreibt - ,im Stillen [mit seinen analytischen Konstruktionen zu beschäftigen und sie primär dazu zu verwenden, die eigene] Einsamkeit zu möblieren und sich zu befreien von [einem] Zuviel, [von einem] Über-schuss an Wissen“"(S. 208). Der Versuch, Patienten zu verstehen - so Borens weiter - lasse den analytischen Diskurs zu einem konventionellen verkommen. Vielmehr sollte es doch in der Analyse vor allem darum gehen, ,das nicht vermeidbare Vorverständnis auf ein Minimum zu reduzieren und ganz besonders den Text sprechen zu lassen“ (Borens 1988, S. 214). Worauf es ankomme sei, mit einer Deutung nichts abzuschließen, nichts zu vollenden, ,, sondern auf etwas Neues, auf etwas Anderes“ (Borens 1988, S. 209), hinzuweisen, etwas zu evozieren. Anhand mehrerer Fallbeispiele demonstriert Borens, dass bspw. die Signifikanz des ,acting out“" in einer Analyse darin besteht, dass es auf sich selbst zeigt und nicht interpretiert werden kann: „Das andere Element der Aktion, nämlich das bezeichnende, weist auf ein unbewusstes Subjekt hin, das in seiner ihm selbst nicht bewussten Aktion sich an den Andern wendet" (2013, S. 179). In eine ganz ähnliche Richtung weisen die Argumente von Pohlen und Bautz-Holzherr (1991), die folglich die Psychoanalyse als eine Lehre vom Finden definieren.

Die Signifikantendeutung ist mithin die notwendig Voraussetzung, um dem Analysanden seine unbewussten Intentionen hörbar zu machen. Das setzt allerdings voraus, dass der Analytiker für sein eigenes Selbstverständnis primär seinen Mangel an Sein und nicht sein (Analytiker-)Sein zugrunde legt.

Open Access Dieser Artikel unterliegt den Bedingungen der Creative Commons Attribution Noncommercial License. Dadurch sind die nichtkommerzielle Nutzung, Verteilung und Reproduktion erlaubt, sofern der/die Originalautor/en und die Quelle angegeben sind.

\section{Literatur}

Bettelheim B (1988) Freud und die Seele des Menschen. Deutscher Taschenbuch Verlag (dtv), München Bollas C (1997) Der Schatten des Objekts. Das ungedachte Bekannte. Zur Psychoanalyse der frühen Entwicklung. Klett-Cotta, Stuttgart

Bollas C (2006) Übertragungsdeutung als ein Widerstand gegen die freie Assoziation. Psyche - Z Psychoanal 60: 932-947

Borens R (1988) Ich weiss nicht, was soll ich denn deuten. RISS 3: 204-214 
Borens R (1993) Psychologisches Verstehen und psychoanalytisches Verständnis. In: Michels A, Widmer P, Müller P (Hrsg) Eine Technik für die Psychoanalyse? Königshausen \& Neumann, Würzburg

Borens R (2013) Der analytische Akt als symbolisches Geschehens. Jb Psychoanal 66: 171-191

Deutscher G (2010) Im Spiegel der Sprache. Warum die Welt in anderen Sprachen anders aussieht. C.H. Beck, München

Falk D, Kuhlmann-Krieg S (2010) Wie die Menschheit zur Sprache fand: Mütter, Kinder und der Ursprung des Sprechens. DVA, München

Fink B (2005) Eine klinische Einführung in die Lacansche Psychoanalyse. Turia + Kant, Wien

Freud S (1895d) Studien über Hysterie. GW Bd. 1, S 75-312

Freud S (1901a) Zur Psychopathologie des Alltagslebens. GW Bd. 4

Freud S (1905a) Über Psychotherapie. GW Bd. 5, S 13-26

Freud S (1910k) Über ,wilde“ Psychoanalyse. GW Bd. 8, S 118-125

Freud S (1912b) Zur Dynamik der Übertragung. GW Bd. 8, S 364-374

Freud S (1912e) Ratschläge für den Arzt bei der psychoanalytischen Behandlung. GW Bd. 8, 376-387

Freud S (1914d) Zur Geschichte der psychoanalytischen Bewegung. GW Bd. 10, S 43-113

Freud S (1916-17a) Vorlesungen zur Einführung in die Psychoanalyse. GW Bd. 11

Freud S (1919a) Wege der psychoanalytischen Therapie. GW Bd. 12, S 183-194

Freud S (1925f) Geleitwort zu: Aichhorn, August, Verwahrloste Jugend. Die Psychoanalyse in der Fürsorgeerziehung. GW Bd. 14, S 565-567

Freud S (1937d) Konstruktionen in der Analyse. GW Bd. 16, S 43-56

Freud S (1940a) Abriß der Psychoanalyse. GW Bd. 17, S 63-138

Freud S (1985c) Briefe an Wilhelm Fließ. Fischer, Frankfurt a. M.

Goldschmidt G-A (1999) Als Freud das Meer sah. Amman, Zürich

Goldschmidt G-A (2006) Freud wartet auf das Wort. Amman, Zürich

Heimann P (1950) Über die Gegenübertragung. Forum Psychoanal 12: 179-184

Kläui C (2002) Gegenübertragung und der Wunsch, Analytiker zu sein. Jb Klin Psychoanal 4: 54-64

Klemann M (2008) „Wer nicht hören will, muss fühlen“ - Übertragungsanalyse und die unbewussten Wünsche des Analytikers. Psyche - Z Psychoanal 62: 397-422

Knellessen O, Passett P, Schneider P (2003) Das Deuten der Psychoanalyse. Turia +Kant, Wien

König K (2007) Transfer - Von der Psychotherapie in den Alltag. Klett-Cotta, Stuttgart

König K (2010) Gegenübertragung und die Persönlichkeit des Psychotherapeuten. Brandes \& Apsel, Frankfurt a. M.

Lacan J (1986) Das Seminar Buch 20 [1972-1973]. Encore. Quadriga, Weinheim-Berlin

Lacan J (1991) Das Seminar Buch 2 [1954-1955]. Das Ich in der Theorie Freuds und in der Technik der Psychoanalyse. Quadriga, Weinheim-Berlin

Lacan J (1996) Die Ausrichtung der Kur und die Prinzipien ihrer Macht [1958]. In: Schriften I: 171-236. Quadriga, Weinheim-Berlin

Mayer A (2002) Mikroskopie der Psyche. Die Anfänge der Psychoanalyse im Hypnose-Labor. Wallstein, Göttingen

Pohlen M, Bautz-Holzherr M (1991) Eine andere Aufklärung. Das Freudsche Subjekt in der Analyse. Suhrkamp, Frankfurt a. M.

Sterba R (1934) Das Schicksal des Ichs im therapeutischen Verfahren. IZ 20: 66-73

Thomä H (2008) Clarissa X. Sackgassen und Behandlungsabbrüche. Psychotherapeut 53: 138-142

Žižek S (1988) Das Subjekt, dem unterstellt wird, zu... RISS 3: 159-171

Manfred Klemann, Jg. 1948, Dr. phil., Dipl.-Psych., Psychoanalytiker, analytischer Paar- und Familientherapeut; in eigener Praxis tätig. Lehranalytiker und Dozent am Lou Andreas-Salomé Institut für Psychoanalyse und Psychotherapie (DPG, VAKJP) Göttingen. Veröffentlichungen zur Familientherapie, Suizidologie und Theoriegeschichte der Psychoanalyse. 